\title{
ImmunoCyt test compared to cytology in the diagnosis of bladder cancer: A meta-analysis
}

\author{
HOUGUANG HE, CONGHUI HAN, LIN HAO and GUANGHUI ZANG \\ The Affiliated Xuzhou Hospital of Medical College of Southeast University, \\ Xuzhou Central Hospital, Xuzhou, Jiangsu 221009, P.R. China
}

Received January 14, 2016; Accepted May 12, 2016

DOI: $10.3892 / \mathrm{ol} .2016 .4556$

\begin{abstract}
The aim of the present study was to evaluate the diagnostic value of the ImmunoCyt test compared with urine cytology in detecting bladder cancer. A systematic literature search was performed to locate all publications reporting on the diagnostic accuracy of the ImmunoCyt test for bladder cancer. Data were extracted from $2 \times 2$ tables or calculated from reported accuracy data. Collected data were meta-analyzed for sensitivity, specificity, positive likelihood ratio (LR), negative LR, diagnostic odds ratio (DOR), and summary receiver operator characteristic (sROC) curve analysis. We applied the Meta-DiSc 1.4 and STATA 13.0 software to the meta-analysis. Seven separate studies consisting of 1,602 patients with bladder cancer were considered in the meta-analysis. We found that the ImmunoCyt test had a higher sensitivity than the urine cytology test $[0.725,95 \%$ confidence interval (CI) $0.683-0.765$ vs. $0.566,95 \%$ CI, $0.521-0.611]$, but the specificity, positive LR, negative LR, DOR, area under the curve (AUC) and Q index of the ImmunoCyt test were lower compared with the urine cytology test. In addition, the pooled sensitivity, specificity, positive LR, negative LR, DOR, AUC, and Q index of the combined method (combination of ImmunoCyt and cytology) were $0.833,0.644,2.804,0.228$, $13.50,0.8554$ and 0.7863 , respectively. The results of the Egger's test showed no publication bias $(\mathrm{P}>0.05)$. In conclusion, specificity, positive LR, negative LR, DOR, the AUC, and the $\mathrm{Q}$ index of the urine cytology test may be superior to the ImmunoCyt test, but the ImmunoCyt test has greater sensitivity than the urine cytology test. Use of ImmunoCyt and cytology in combination has the potential to improve the sensitivity and promises to be an alternative in the detection of bladder cancer.
\end{abstract}

Correspondence to: Dr Houguang He, The Affiliated Xuzhou Hospital of Medical College of Southeast University, Xuzhou Central Hospital, 199 Jiefang South Road, Xuzhou, Jiangsu 221009, P.R. China

E-mail:vfikr493121@163.com

Key words: bladder cancer, ImmunoCyt test, cytology

\section{Introduction}

Bladder cancer, the most common malignancy of the urinary tract, poses a threat to an increasing number of humans (1). Progression of bladder tumors greatly increases the risk of metastasis and subsequent mortality. Consequently, early detection is crucial for improving patient prognosis and long-term survival.

Currently, cystoscopy is considered the gold standard for the detection of primary tumors and also the follow-up of patients after transurethral resection of bladder tumors, despite being an invasive approach (2). Urine cytology, another widely used detection method, has been firmly established as a useful adjunct, not only in the diagnosis, but also the follow-up of patients with bladder cancer (3). However, it is highly grade-dependent, and in low-grade cancers, it seems to not be sensitive enough. In addition, urine cytology also suffers from a high inter- and intra-observer variability, which limits its validity in screening for bladder tumors $(4,5)$.

With the aim of improving the lower sensitivity of cytology and increasing the detection of low-grade tumors, the $\mathrm{uCyt}+/$ ImmunoCyt test was developed by Fradet and Lockhard, it uses three monoclonal antibodies to detect urothelial cells in voided urine (6). A number of previous studies demonstrated its high sensitivity, and some investigators suggested that it also plays an important role in predicting urothelial tumor recurrence (7-11). The uCyt+/ImmunoCyt test has been in limited clinical and research use for a decade, but its diagnostic accuracy has never been subjected to a quantitative review. We therefore performed a systematic review and meta-analysis of uCyt+/ImmunoCyt test to fully assess its diagnostic accuracy in detecting bladder cancers and to guide future implementation.

\section{Materials and methods}

Search methodology. We searched Medline and PubMed for articles that reported on the diagnostic sensitivity and specificity of the uCyt+/ImmunoCyt test for bladder cancers. The keywords used for the search were: (uCyt+/ImmunoCyt OR ImmunoCyt), (bladder cancer or urothelial tumor), sensitivity, specificity, and cytology. Only data from original published papers were collected, with meeting or conference abstracts being excluded. Primary sources from tracking references 
Table I. Characteristics of studies included in the meta-analysis $(n=7)$.

\begin{tabular}{|c|c|c|c|c|c|c|c|c|c|c|c|c|c|c|c|}
\hline \multirow[b]{2}{*}{ Author (refs.) } & \multirow[b]{2}{*}{ Year } & \multirow{2}{*}{$\begin{array}{c}\text { Sample } \\
\text { size }\end{array}$} & \multirow{2}{*}{$\begin{array}{l}\text { STARD } \\
\text { score }\end{array}$} & \multicolumn{4}{|c|}{ uCyt+/ ImmunoCyt } & \multicolumn{4}{|c|}{ Cytology } & \multicolumn{4}{|c|}{ Combination of both } \\
\hline & & & & $\mathrm{TP}$ & FP & $\mathrm{FN}$ & $\mathrm{TN}$ & $\mathrm{TP}$ & $\mathrm{FP}$ & $\mathrm{FN}$ & $\mathrm{TN}$ & $\mathrm{TP}$ & FP & $\mathrm{FN}$ & $\mathrm{TN}$ \\
\hline Yafi et al (18) & 2014 & 109 & 18 & 52 & 5 & 31 & 21 & 36 & 4 & 47 & 22 & 54 & 6 & 29 & 20 \\
\hline Soyuer et al (19) & 2009 & 90 & 15 & 45 & 5 & 9 & 31 & 41 & 12 & 13 & 24 & 48 & 5 & 6 & 31 \\
\hline Horstmann et al (20) & 2009 & 221 & 16 & 82 & 30 & 31 & 78 & 95 & 41 & 18 & 67 & 105 & 47 & 8 & 61 \\
\hline Têtu et al (7) & 2005 & 870 & 19 & 100 & 281 & 36 & 453 & 39 & 17 & 97 & 717 & 114 & 284 & 22 & 450 \\
\hline Toma et al (21) & 2004 & 120 & 15 & 33 & 20 & 9 & 58 & 36 & 16 & 6 & 62 & 37 & 21 & 5 & 57 \\
\hline Hautmann et al (22) & 2004 & 94 & 14 & 19 & 16 & 11 & 48 & 22 & 13 & 8 & 51 & 25 & 9 & 5 & 55 \\
\hline Sullivan et al (11) & 2009 & 98 & 18 & 20 & 26 & 6 & 46 & 5 & 2 & 21 & 70 & 20 & 26 & 6 & 46 \\
\hline
\end{tabular}

TP, true positive; FP, false positive; FN, false negative; TN, true negative; STARD, Standards for the Reporting of Diagnostic Accuracy Studies.

were also obtained from manual searches in review papers and original articles. Electronic databases were retrieved by two independent investigators. If the assessments of the investigators were not consistent, a discussion ensued whether to include the data or not. The articles included contained studies of the patients with bladder cancer diagnosed using the $\mathrm{uCyt+/ImmunoCyt} \mathrm{and} \mathrm{cytology} \mathrm{tests.} \mathrm{The} \mathrm{effect} \mathrm{sizes} \mathrm{of}$ the studies seen as odds ratio (OR), sample size, gender, or range of age did not exclude any articles. Studies that only described the uCyt+/ImmunoCyt test or cytology data were excluded.

Data extraction. Test performance data were extracted as a 2x2 table of true-positive (TP), false-positive (FP), false-negative $(\mathrm{FN})$ and true-negative $(\mathrm{TN})$ values directly from tabulated results (Table I). If these were not directly available, they were calculated from reported sensitivity, specificity, positive predictive value (PPV) and/or negative PV (NPV).

Data analysis. The estimates of sensitivity, specificity, likelihood ratios (LRs), diagnostic odds ratio (DOR), and their 95\% confidence intervals (95\% CIs) were pooled for each study. The within- and between-study variation or heterogeneity were assessed by testing Cochran's Q-statistic. Heterogeneity was calculated using the formula $\mathrm{I}_{2}=100 \% \mathrm{x}(\mathrm{Q}-\mathrm{df}) / \mathrm{Q}(12)$. A significant $\mathrm{Q}$-statistic $(\mathrm{P}<0.10)$ or $\mathrm{I}_{2}$-statistic $\left(\mathrm{I}_{2}>50\right)$ indicated heterogeneity across studies, and then the random effect model (REM) was used; otherwise, the fixed effect model (FEM) was used. The pooled estimate of ORs was obtained using the Mantel and Haenszel method for FEM, the DerSimonian and Laird and DerSimonian and Kacker method for REM (13-15).

The summary receiver operator characteristic (sROC) curve was used to graphically determine performance following testing for correlation between sensitivity and specificity [as the logit TP rate (TPR) vs. the logit FP rate (FPR)] (16).

The area under the curve (AUC) and an index $\mathrm{Q}$ were discussed as potentially useful summaries of the curve. An upper bound was derived for the AUC based on an exact analytic expression for the homogeneous situation, and a

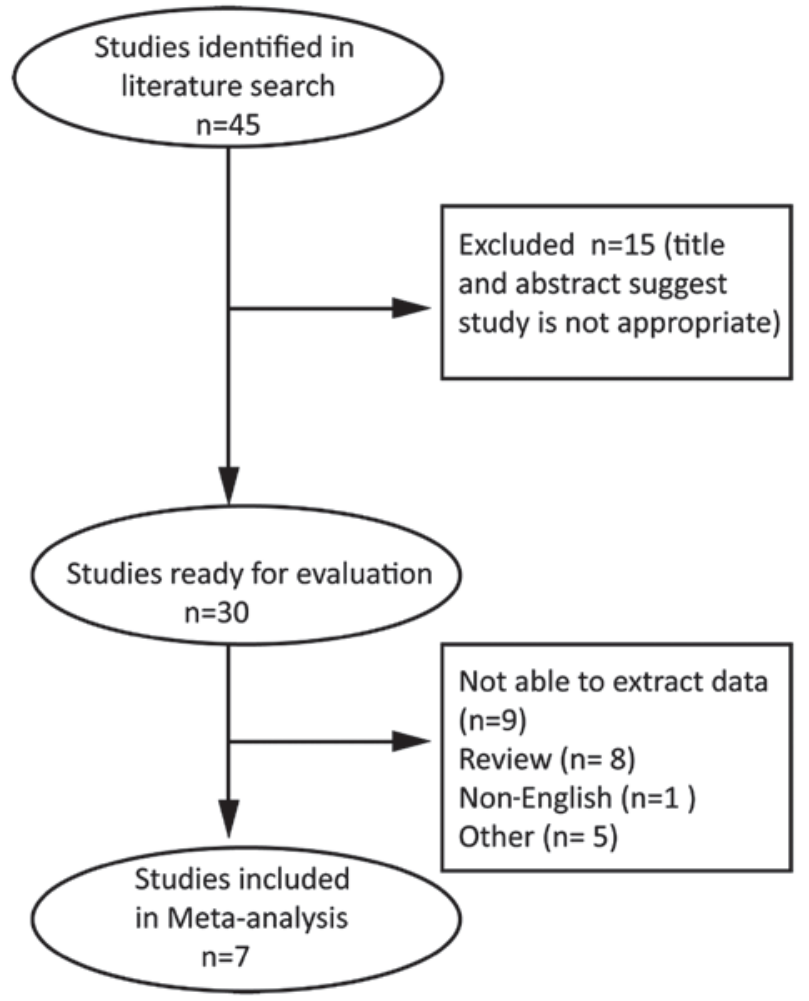

Figure 1. Flow diagram for selection of studies for the meta-analysis.

lower bound based on the limit case Q, defined by the point where sensitivity equals specificity: $\mathrm{Q}$ is invariant to heterogeneity (17).

Analyses were performed using the freeware Meta-Disc version 1.4 (Universidad Complutense, Madrid, Spain) and STATA package version 13.0 (Stata Corporation, College Station, TX, USA). All P-values were two-sided. $\mathrm{P}<0.05$ was considered to indicate a statistically significant difference.

\section{Results}

In total, 45 potentially relevant studies were identified (Fig. 1). During the abstract screening, 15 articles were excluded. 
Table II. The indices of bladder cancer diagnosed by ImmunoCyt test and cytology test.

\begin{tabular}{|c|c|c|c|c|c|c|c|c|c|}
\hline \multirow{2}{*}{$\begin{array}{l}\text { Diagnostic } \\
\text { methods }\end{array}$} & \multirow[b]{2}{*}{ Parameter } & \multicolumn{2}{|c|}{ Test of association } & \multicolumn{3}{|c|}{ Test of heterogeneity } & \multirow[b]{2}{*}{ Model } & \multicolumn{2}{|c|}{$\begin{array}{l}\text { Egger's test for } \\
\text { publication bias }\end{array}$} \\
\hline & & Estimates & $95 \% \mathrm{CI}$ & Q & P-value & $\mathrm{I}_{2}(\%)$ & & $\mathrm{t}$ & P-value \\
\hline \multirow[t]{5}{*}{ uCyt+/ImmunoCyt } & Sensitivity & 0.725 & $0.683-0.765$ & 9.66 & 0.140 & 37.9 & FEM & 2.56 & 0.051 \\
\hline & Specificity & 0.657 & $0.629-0.685$ & 23.23 & 0.001 & 74.2 & REM & & \\
\hline & Positive LR & 2.578 & $2.003-3.318$ & 17.56 & 0.007 & 65.8 & REM & & \\
\hline & Negative LR & 0.385 & $0.327-0.452$ & 8.43 & 0.208 & 28.9 & FEM & & \\
\hline & DOR & 7.114 & $4.709-10.748$ & 11.24 & 0.081 & 46.6 & REM & & \\
\hline \multirow[t]{5}{*}{ Cytology } & Sensitivity & 0.566 & $0.521-0.611$ & 132.48 & 0.000 & 95.5 & REM & -0.72 & 0.505 \\
\hline & Specificity & 0.906 & $0.887-0.923$ & 161.32 & 0.000 & 96.3 & REM & & \\
\hline & Positive LR & 3.862 & $2.347-6.353$ & 36.95 & 0.000 & 83.8 & REM & & \\
\hline & Negative LR & 0.459 & $0.320-0.658$ & 66.46 & 0.000 & 91.0 & REM & & \\
\hline & DOR & 10.269 & $7.501-15.795$ & 8.77 & 0.187 & 31.6 & FEM & & \\
\hline \multirow{5}{*}{$\begin{array}{l}\text { Combination } \\
\text { of both }\end{array}$} & Sensitivity & 0.833 & $0.796-0.865$ & 28.17 & 0.000 & 78.7 & REM & 1.80 & 0.132 \\
\hline & Specificity & 0.644 & $0.615-0.672$ & 34.10 & 0.000 & 82.4 & REM & & \\
\hline & Positive LR & 2.804 & $2.163-3.636$ & 23.59 & 0.001 & 74.6 & REM & & \\
\hline & Negative LR & 0.228 & $0.149-0.350$ & 21.42 & 0.002 & 72.0 & REM & & \\
\hline & DOR & 13.50 & $7.847-23.238$ & 14.56 & 0.024 & 58.8 & REM & & \\
\hline
\end{tabular}

CI, confidence interval; LR, likelihood ratio; DOR, diagnostic odds ratio; FEM, fixed effect model; REM, random effect model.

Thirty studies were considered for full publication review. Of these, 23 were excluded (nine because there were no data available, eight because they were reviews, one because it was a non-English article, five because they only included cytology or ImmunoCyt data and no comparison). Seven studies were eventually analyzed (Table I) $(7,11,18-22)$. These studies were published between 2004 and 2014, and included a total of 1,602 patients with bladder cancer. Their sample size ranged from 90 to 870 . In these seven studies, a combination of cystoscopy and biopsy was used as the gold standard of bladder cancer.

Assessment of study quality. Studies meeting the criteria were quality assessed using positive scoring in a modified 23-point Standards for the Reporting of Diagnostic Accuracy Studies (STARD) proforma $(23,24)$. Two readers (F.T. and M.M.) independently assessed the included studies according to the prearranged proforma. An open discussion was subsequently held in order to resolve any disagreement between the readers. STARD scores for each study are provided in Table I.

Pooled sensitivity, specificity, $L R+, L R-$, and DOR. We summarized the overall meta-analysis of bladder cancer patients with the ImmunoCyt test, cytology test, and combined method (Combination of ImmunoCyt and Cytology) (Table II). The REM or FEM was used to combine the data of TP, FP, FN, and TN numbers.

ImmunoCyt test. The pooled sensitivity of the ImmunoCyt test for all seven studies included in the final meta-analysis was $72.5 \%$ (95\% CI, 68.3-76.5\%), the pooled specificity $65.7 \%$ (95\% CI, 62.9-68.5\%), the pooled positive LR was 2.578 (95\% CI, 2.003-3.318), the pooled negative LR was 0.385 (95\% CI, 0.327-0.452), and the pooled DOR was 7.114 (95\% CI, 4.709-10.748) (Table II).

Cytology test. The pooled sensitivity of cytology test was $56.6 \%$ (95\% CI, 52.1-61.1\%), the pooled specificity was $90.6 \%$ (95\% CI, 88.7-92.3\%), the pooled positive LR was 3.862 (95\% CI, 2.347-6.353), the pooled negative LR was 0.459 (95\% CI, 0.320-0.658), and the pooled DOR was 10.269 (95\% CI, 7.501-15.795) (Table II).

Combined method (ImmunoCyt and cytology). We also collected the pooled meta-analysis of bladder cancer patients with the combined method. The pooled sensitivity was $83.3 \%$ (95\% CI, 79.6-86.5\%), the pooled specificity was $64.4 \%$ (95\% CI, 61.5-67.2\%), the pooled positive LR was 2.804 (95\% CI, 2.163-3.636), the pooled negative LR was 0.228 (95\% CI, 0.149-0.350), and the pooled DOR was 13.50 (95\% CI, 7.847-23.238) (Table II).

$A U C$ and $Q$ index in the three tests. The AUC and Q index of ImmunoCyt test were 0.7910 and 0.7280 , those of the cytology test 0.8239 and 0.7570 , and those of the combined method 0.8554 and 0.7863 , respectively (Figs. 2-4).

Publication bias. Egger's test was used to assess the publication bias. For all samples, Egger's test provided no evidence of publication bias for this meta-analysis. Detailed information is provided in Fig. 5. 


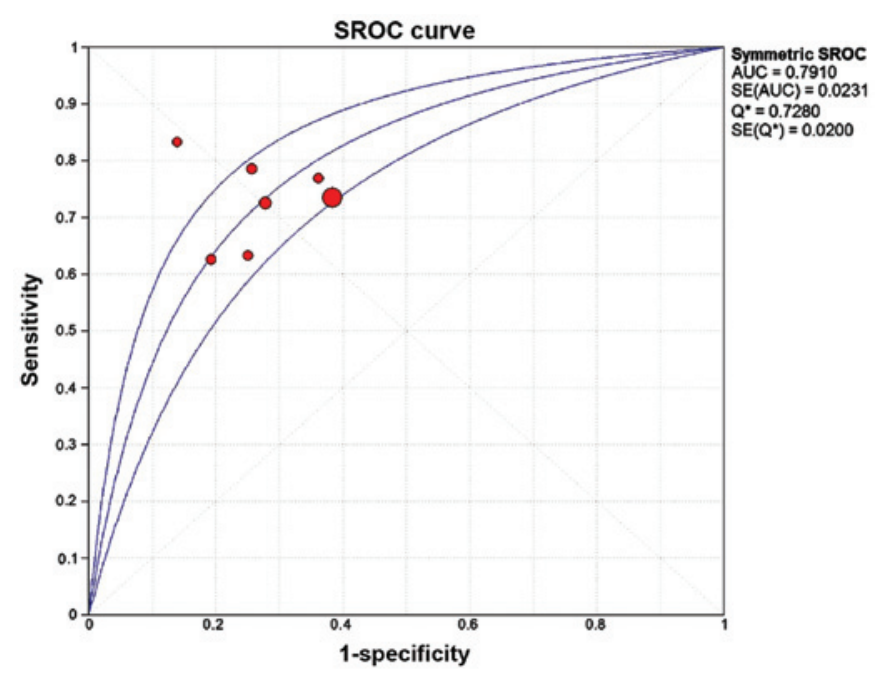

Figure 2. The summary receiver operating characteristic (sROC) curve of ImmunoCyt test.

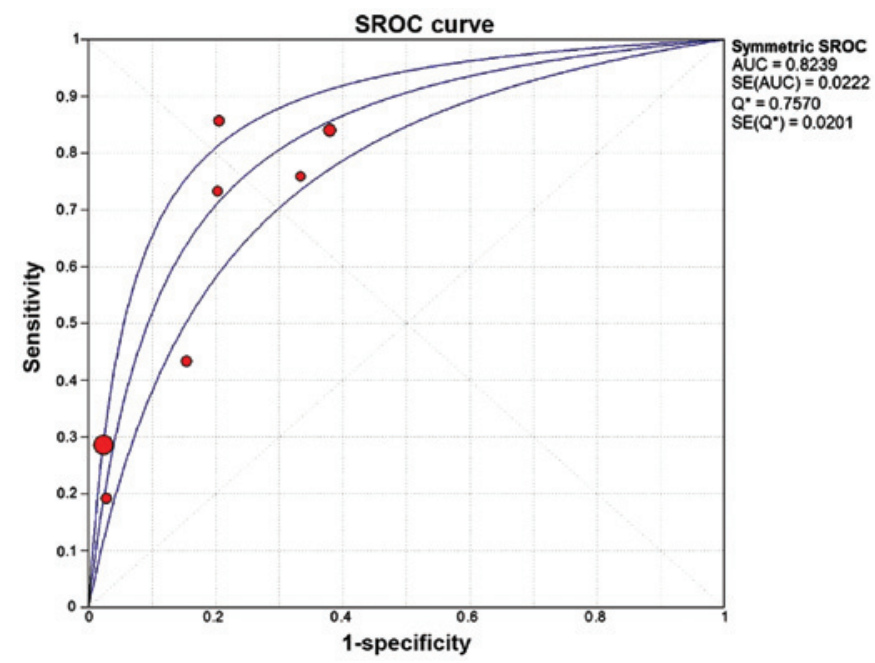

Figure 3. The summary receiver operating characteristic (sROC) curve of cytology test.

\section{Discussion}

As a useful adjunct for cystoscopy, cytology has been used for more than 60 years for the diagnosis of urothelial carcinoma (UC). The test suffers from low sensitivity (38-51\%), but boasts a high specificity (94-98\%), particularly for high-grade disease (25). The reliability of cytology results depends on sample quality and cytopathologist experience to some extent. Atypical results are often found in urine cytology tests (6). Among these atypical cytology results, biopsy reveals that malignancy underlies $23-68 \%$ of them. Investigators have also found that its sensitivity is highly associated with tumor stage. For patients with higher-grade bladder cancer, it appears to be more sensitive (26-29).

With the aim of improving the low sensitivity of cytology and the detection of low-risk UC, many voided urine biomarker assays such as UroVysion, NMP22, BTA TRAK, and ImmunoCyt/uCyt+ have been developed but have yet to gain widespread clinical application. The ImmunoCyt test

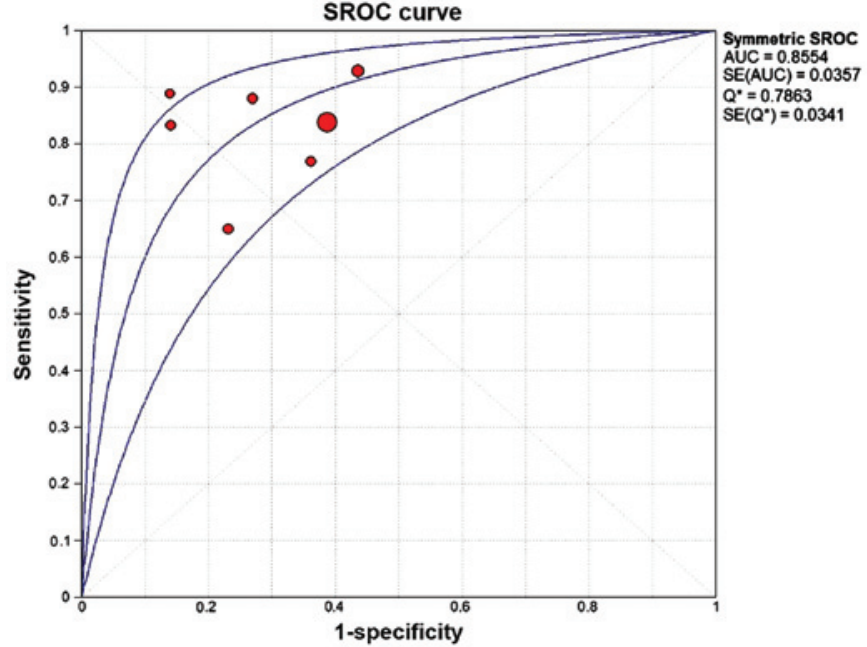

Figure 4. The summary receiver operating characteristic (sROC) curve of combined test.

performance has been well studied since its introduction by Fradet and Lockhard in 1997 (6,30). It is a triple immunofluorescent monoclonal antibody assay associated with UC, approved by the US Food and Drug Administration. A previous series reported a sensitivity of $74-87 \%$ and specificity of 62-78\%, with PPVs and NPVs of 26-67\% and 91-96\%, respectively (6).

Additionally, the ImmunoCyt test can also provide important information in evaluating hematuria, particularly in patients with negative imaging and cystoscopy but atypical cytology (31).

Many studies have reported on the diagnostic accuracy of the ImmunoCyt and cytology tests in detecting bladder cancer (29-32). The results of those studies, however, have been met with skepticism due to their small sample sizes or low statistical power. In the present meta-analysis, we combined seven separate studies, consisting of 1,602 patients to compare the diagnostic accuracy of the ImmunoCyt test with the urine cytology test in detecting bladder cancer. We found that the ImmunoCyt test had a higher sensitivity than the urine cytology test, but the specificity, positive LR, negative LR, and DOR of the ImmunoCyt test were lower compared with the urine cytology test. In addition, the AUC and Q index of cytology were superior to those of the ImmunoCyt test. Based on these comparisons, we can conclude that the ImmunoCyt test would not replace the urine cytology test in detecting bladder cancer.

We also collected data of a combined method (combination of ImmunoCyt test and cytology). The pooled sensitivity, specificity, positive LR, negative LR, DOR, AUC, and Q index were $0.833,0.644,2.804,0.228,13.50$, 0.8554 and 0.7863 , respectively. These data suggest that the ability of cytology to detect malignancies of the bladder can be highly improved in combination with ImmunoCyt. The results of the present study are in agreement with previous findings of Mian et al regarding the clinical usefulness of combining the two tests (32). Using the uCyt1/ImmunoCyt test in combination with cytology to detect bladder cancer appeared to improve the overall sensitivity for cytology 



\begin{tabular}{|c|c|c|c|c|c|c|}
\hline \multirow{2}{*}{$\frac{\text { Egger's test }}{\text { Std_Eff }}$} & \multicolumn{2}{|c|}{ ImmunoCyt } & \multirow[b]{2}{*}{$\mathrm{t}$} & \multirow[b]{2}{*}{$P>|t|$} & \multirow{2}{*}{\multicolumn{2}{|c|}{ [95\% Conf. Interval] }} \\
\hline & Coef. & Std. Err. & & & & \\
\hline slope & 1.397798 & 0.1245427 & 11.22 & 0.000 & 1.07765 & 1.717945 \\
\hline bias & 3.241132 & 1.265997 & 2.56 & 0.051 & -0.0132164 & 6.49548 \\
\hline \multicolumn{7}{|c|}{ Cytology } \\
\hline Std_Eff & Coef. & Std. Err. & $\mathrm{t}$ & $P>|t|$ & \multicolumn{2}{|c|}{ [95\% Conf. Interval] } \\
\hline slope & 2.667106 & 0.3435523 & 7.76 & 0.001 & 1.783977 & 3.550235 \\
\hline bias & -1.488471 & 2.0746 & -0.72 & 0.505 & -6.821401 & 3.844459 \\
\hline Egger's test & \multicolumn{2}{|c|}{ Combination } & & & & \\
\hline Std_Eff & Coef. & Std. Err. & $\mathrm{t}$ & $P>|t|$ & \multicolumn{2}{|c|}{ [95\% Conf. Interval] } \\
\hline slope & 1.949838 & 0.2083499 & 9.36 & 0.000 & 1.414257 & 2.485418 \\
\hline bias & 2.696009 & 1.500989 & 1.80 & 0.132 & -1.162407 & 6.554425 \\
\hline
\end{tabular}

Figure 5. Egger's publication bias plots.

alone. Furthermore, the use of the two tests in combination constitute a potential alternative option to limit the amount of cystoscopic evaluations required for the follow-up of patients with low-risk bladder cancer.

One limitation of our study is heterogeneity. The considerable amount of heterogeneity detected between the studies suggests a need for caution when pooling the diagnostic accuracy measures together. Additionally, between-study heterogeneities may distort the meta-analysis. The degree of heterogeneity is one of the major concerns in a meta-analysis, as non-homogeneous data are liable to result in misleading results (33). Different populations also contribute to the heterogeneity. Therefore, the results of this meta-analysis should be interpreted with caution. We minimized the likelihood of bias by developing a detailed protocol prior to initiating the study, by performing a rigorous search of published studies and by using explicit methods for study selection, data extraction, and data analysis.

Another limitation common to diagnostic meta-analyses is the lack of clarity, quality, and standardization in the methodology of diagnostic studies. Studies were assessed for quality using STARD proforma to quantify the methodology of the study design. Consequently, we excluded certain studies due to ambiguity between the raw data and the diagnostic accuracy data in the process of data extraction.

In conclusion, in this meta-analysis, we have evaluated the pooled sensitivity, specificity, positive LR, negative LR, DOR, AUC, and Q index of three tests (ImmunoCyt, cytology, and combined test) from seven studies. We found that ImmunoCyt was superior to cytology only in sensitivity, however, inferior to cytology in specificity, positive LR, negative LR, DOR, AUC, and Q index. In conclusion, cytology remains integral in the detection of bladder cancer. Meta-analysis of the combined tests suggests that the use of ImmunoCyt and cytology in combination significantly improves the sensitivity for detecting bladder cancer and promises to be an alternative option in the clinical setting.

\section{References}

1. Ploeg M, Aben KK and Kiemeney LA: The present and future burden of urinary bladder cancer in the world. World J Urol 27: 289-293, 2009.

2. Garfield SS, Gavaghan MB, Armstrong SO and Jones JS: The cost-effectiveness of blue light cystoscopy in bladder cancer detection: United States projections based on clinical data showing 4.5 years of follow up after a single hexaminolevulinate hydrochloride instillation. Can J Urol 20: 6682-6689, 2013.

3. Sullivan PS, Chan JB, Levin MR and Rao J: Urine cytology and adjunct markers for detection and surveillance of bladder cancer. Am J Transl Res 2: 412-440, 2010.

4. Talwar R, Sinha T, Karan SC, Doddamani D, Sandhu A, Sethi GS, Srivastava A, Narang V, Agarwal A and Adhlakha N: Voided urinary cytology in bladder cancer: is it time to review the indications? Urology 70: 267-271, 2007.

5. Wiener HG: Cytology of the urinary tract. A modern investigation method? Pathologe 28: 354-359, 2007 (In German).

6. Fradet $Y$ and Lockhard C: Performance characteristics of a new monoclonal antibody test for bladder cancer: ImmunoCyt trade mark. Can J Urol 4: 400-405, 1997. 
7. Têtu B, Tiguert R, Harel F and Fradet Y: ImmunoCyt/uCyt+ improves the sensitivity of urine cytology in patients followed for urothelial carcinoma. Mod Pathol 18: 83-89, 2005.

8. Messing EM, Teot L, Korman H, Underhill E, Barker E, Stork B, Qian J and Bostwick DG: Performance of urine test in patients monitored for recurrence of bladder cancer: a multicenter study in the United States. J Urol 174: 1238-1241, 2005.

9. Mian C, Maier K, Comploj E, Lodde M, Berner L, Lusuardi L, Palermo S, Vittadello F and Pycha A: uCyt+/ImmunoCyt in the detection of recurrent urothelial carcinoma: an update on 1991 analyses. Cancer 108: 60-65, 2006.

10. Lodde M, Mian C, Comploj E, Palermo S, Longhi E, Marberger M and Pycha A: uCyt+ test: alternative to cystoscopy for less-invasive follow-up of patients with low risk of urothelial carcinoma. Urology 67: 950-954, 2006.

11. Sullivan PS, Nooraie F, Sanchez H, Hirschowitz S, Levin M, Rao PN and Rao J: Comparison of ImmunoCyt, UroVysion, and urine cytology in detection of recurrent urothelial carcinoma: a 'split-sample' study. Cancer 117: 167-173, 2009.

12. Higgins JP, Thompson SG, Deeks JJ and Altman DG: Measuring inconsistency in meta-analyses. BMJ 327: 557-560, 2003.

13. Mantel N and Haenszel W: Statistical aspects of the analysis of data from retrospective studies of disease. J Natl Cancer Inst 22 719-748, 1959.

14. DerSimonian R and Laird N: Meta-analysis in clinical trials. Control Clin Trials 7: 177-188, 1986.

15. DerSimonian R and Kacker R: Random-effects model for meta-analysis of clinical trials: an update. Contemp Clin Trials 28: 105-114, 2007.

16. Walter SD: Properties of the summary receiver operating characteristic (SROC) curve for diagnostic test data. Stat Med 21: 1237-1256, 2002.

17. Altman DG and Bland JM: Diagnostic tests 3: receiver operating characteristic plots. BMJ 309: 188, 1994.

18. Yafi FA, Brimo F, Steinberg J, Aprikian AG, Tanguay S and Kassouf W: Prospective analysis of sensitivity and specificity of urinary cytology and other urinary biomarkers for bladder cancer. Urol Oncol 33: 66.e25-66.e31, 2015.

19. Soyuer I, Sofikerim M, Tokat F, Soyuer S and Ozturk F: Which urine marker test provides more diagnostic value in conjunction with standard cytology- ImmunoCyt/uCyt+ or Cytokeratin 20 expression. Diagn Pathol 4: 20, 2009.

20. Horstmann M, Patschan O, Hennenlotter J, Senger E, Feil G and Stenzl A: Combinations of urine-based tumour markers in bladder cancer surveillance. Scand J Urol Nephrol 43: 461-466, 2009.

21. Toma MI,Friedrich MG, Hautmann SH, Jäkel KT, Erbersdobler A, Hellstern A and Huland H: Comparison of the ImmunoCyt test and urinary cytology with other urine tests in the detection and surveillance of bladder cancer. World J Urol 22: 145-149, 2004.
22. Hautmann S, Toma M, Lorenzo Gomez MF, Friedrich MG, Jaekel T, Michl U, Schroeder GL, Huland H, Juenemann KP and Lokeshwar VB: Immunocyt and the HA-HAase urine tests for the detection of bladder cancer: a side-by-side comparison. Eur Urol 46: 466-471, 2004.

23. Bossuyt PM, Reitsma JB, Bruns DE, Gatsonis CA, Glasziou PP, Irwig LM, Moher D, Rennie D, de Vet HC and Lijmer JG; Standards for Reporting of Diagnostic Accuracy: The STARD statement for reporting studies of diagnostic accuracy: explanation and elaboration. Clin Chem 49: 7-18, 2003.

24. Bossuyt PM, Reitsma JB, Bruns DE, Gatsonis CA, Glasziou PP, Irwig LM, Lijmer JG, Moher D, Rennie D and de Vet HC; STARD Group: Towards complete and accurate reporting of studies of diagnostic accuracy: the STARD initiative. Fam Pract 21: 4-10, 2004.

25. Raab SS, Grzybicki DM, Vrbin CM and Geisinger KR: Urine cytology discrepancies: frequency, causes, and outcomes. Am J Clin Pathol 127: 946-953, 2007.

26. Deshpande V and McKee GT: Analysis of atypical urine cytology in a tertiary care center. Cancer 105: 468-475, 2005.

27. Mokhtar GA, Al-Dousari M and Al-Ghamedi D: Diagnostic significance of atypical category in the voided urine samples: a retrospective study in a tertiary care center. Urol Ann 2: 100-106, 2010.

28. Kapur U, Venkataraman G and Wojcik EM: Diagnostic significance of 'atypia' in instrumented versus voided urine specimens. Cancer 114: 270-274, 2008.

29. Odisho AY, Berry AB, Ahmad AE, Cooperberg MR, Carroll PR and Konety BR: Reflex ImmunoCyt testing for the diagnosis of bladder cancer in patients with atypical urine cytology. Eur Urol 63: 936-940, 2013.

30. Vriesema JL, Atsma F, Kiemeney LA, Peelen WP, Witjes JA and Schalken JA: Diagnostic efficacy of the ImmunoCyt test to detect superficial bladder cancer recurrence. Urology 58: 367-371, 2001

31. Schmitz-Dräger BJ, Beiche B, Tirsar LA, Schmitz-Dräger C, Bismarck E and Ebert T: Immunocytology in the assessment of patients with asymptomatic microhaematuria. Eur Urol 51: 1582-1588, 2007.

32. Mian C, Lodde M, Comploj E, Palermo S, Mian M, Maier K and Pycha A: The value of the ImmunoCyt/uCyt+ test in the detection and follow-up of carcinoma in situ of the urinary bladder. Anticancer Res 25: 3641-3644, 2005.

33. Moreno SG, Sutton AJ, Thompson JR, Ades AE, Abrams KR and Cooper NJ: A generalized weighting regression-derived meta-analysis estimator robust to small-study effects and heterogeneity. Stat Med 31: 1407-1417, 2012. 